Doug Geisler, Eva K. Grebel, and Dante Minniti, eds.

\title{
A New Method for Measuring the Detailed Chemical Composition of Globular Clusters from High-Resolution, Integrated-Light Spectra
}

\author{
Rebecca A. Bernstein \& Andrew McWilliam \\ Carnegie Observatories, 813 Santa Barbara St., Pasadena, CA, 91101
}

\begin{abstract}
We are developing a method for measuring the detailed chemical composition and evolutionary history of extragalactic star clusters from high resolution spectra of their integrated light as one would from spectra of individual stars. In this paper, we show high signal-tonoise ratio echelle spectra of the integrated light of two Galactic globular clusters and equivalent-quality spectra of individual stars in those clusters in order to briefly illustrate some subtleties of the analysis method.
\end{abstract}

\section{Galactic Globular Clusters: A Training Set}

Globular clusters (GCs) have typical velocity dispersions $\left(\sigma_{v}\right)$ in the range 2$20 \mathrm{~km} \mathrm{~s}^{-1}$ (see Figure 1), which is low enough that even the weak absorption features from $\alpha$-elements $(\mathrm{Ca}, \mathrm{O}, \mathrm{Mg}, \mathrm{Ti}, \mathrm{Si}$ ) and many other species (e.g., $\mathrm{Eu}$, $\mathrm{Ba}, \mathrm{Mn}, \mathrm{Fe}$ ) can be measured in the integrated-light spectra. To develop an analysis method which allows us to infer detailed chemical abundances from the ensemble cluster light, we have used the du Pont $2.5 \mathrm{~m}$ telescope at Las Campanas Observatory to obtain integrated-light spectra of 8 Galactic GCs as well as spectra of 3-4 individual stars in those clusters. By comparing the abundances inferred from the integrated light analysis with that from the individual stars, we can use the Galactic GCs as a training set for our technique.

We obtain integrated-light spectra by scanning the telescope uniformly across the inner $32 \times 32$ sq. arcsec region of each Galactic GC. Integration times are 3-5 hrs. In Figure 2 we show an integrated-light spectrum of 47 Tuc and an RGB star in that cluster. Line depths are shallower in the integrated-light spectrum due to the combined effects of velocity dispersion and dilution from the non-RGB cluster population. At the low resolutions typically used for population synthesis studies, none of these lines would have been visible.

\section{Analysis Methods for Integrated-Light Spectra.}

To analyze the integrated-light spectra of Galactic GCs, we synthesize each spectral line for stars over the full range of stellar types contributing to the region of the cluster we observe. We then combine the synthetic spectra in the appropriate mix, as determined from the known color magnitude diagrams (see Figure 3). We compute the synthetic spectra using Kurucz (1993) models and the synthesis program MOOG (Sneden 1974). 
In future studies of distant GCs, where CMDs are not available, we will employ diagnostics derived from the spectra to constrain the mix of stellar populations present in the clusters. For example, the fraction of blue horizontal branch (BHB) stars is indicated by the Balmer-line profiles (see Figure 4). Other diagnostics include the strength of $\mathrm{Fe}$ features as a function of wavelength and the ratios of neutral to ionized line strengths. These lines indicate the numbers of hot BHB stars relative to cool stars and giants relative to dwarfs.

As an initial demonstration of our analysis method, we have determined abundances for NGC 6397 from its integrated light by matching the measured equivalent widths of many weak absorption lines (15 to $100 \mathrm{~m} \AA$ ) with theoretical equivalent widths calculated for different abundances. As shown in the table, our results agree well with those in the literature derived from individual giant stars in NGC 6397.

\begin{tabular}{|lccccc|c|}
\hline Species, X & $\epsilon(X)^{a}$ & $N_{\text {lines }}$ & $\sigma$ & $\sigma / \sqrt{N}$ & {$[X / \mathrm{Fe}]^{b}$} & {$[X / \mathrm{Fe}]^{c}$} \\
\hline Fe I & 5.53 & 26 & 0.29 & 0.06 & -1.97 & -2.00 \\
Fe II & 5.30 & 3 & 0.37 & 0.22 & -2.20 & $\ldots$ \\
Mg I & 5.58 & 1 & $\ldots$ & $\ldots$ & -0.03 & $\ldots$ \\
Ca I & 5.02 & 8 & 0.36 & 0.13 & +0.64 & +0.20 \\
Ti II & 3.33 & 3 & 0.17 & 0.10 & +0.36 & +0.43 \\
Ba II & 0.35 & 3 & 0.23 & 0.13 & +0.10 & -0.16 \\
\hline
\end{tabular}

${ }^{a}$ The abundance of element $X$ is defined as $\epsilon(X)=\log _{10}[\mathrm{~N}(\mathrm{X}) / \mathrm{N}(\mathrm{H})]+12$.

${ }^{b}[\mathrm{X} / \mathrm{Fe}]=\epsilon(\mathrm{X} / \mathrm{Fe})-\epsilon(\mathrm{X} / \mathrm{Fe})_{\odot}$. For $\mathrm{Fe} \mathrm{I}$ and $\mathrm{Fe}$ II this column indicates $[\mathrm{Fe} / \mathrm{H}]$.

$c$ From Castilho et al. 2000.

Note: As the abundances are likely affected by systematic errors, the true uncertainty lies between the $r m s$ error, $(\sigma)$, and the error in the mean, $(\sigma / \sqrt{N})$.

\section{Applications and Upcoming Work}

We have demonstrated that high-resolution, integrated-light spectra can be used to extract element abundances of GCs, with accuracies comparable to those currently obtainable for individual red giant stars. For the brightest GCs, with $M_{\mathrm{v}} \sim-10$, detailed chemical abundances could be measured to $\sim 6 \mathrm{Mpc}$.

As the first application of this method, we have obtained integrated-light spectra of 10 LMC clusters to determine their evolutionary histories and test the standard paradigm for chemical evolution for the LMC.

\section{References}

Castilho, B, Pasquini, L, Allen, D, Barbuy, B, Molaro, P 2000, A\&A, 361, 92 Kaluzny, J. 1997, A\&AS, 122, 1

Kurucz, R.L. 1993, CD-ROM 1-23 (Cambridge: Smithsonian Astrophys. Obs.) Pryor, C. \& Meylan, G. 1993, in Structure and Dynamics of Globular Clusters, ASP Conference Series V. 50, eds. S.G. Djorgovski and G. Meylan

Sneden, C. 1974, PhD Thesis, Univ. of Texas at Austin 


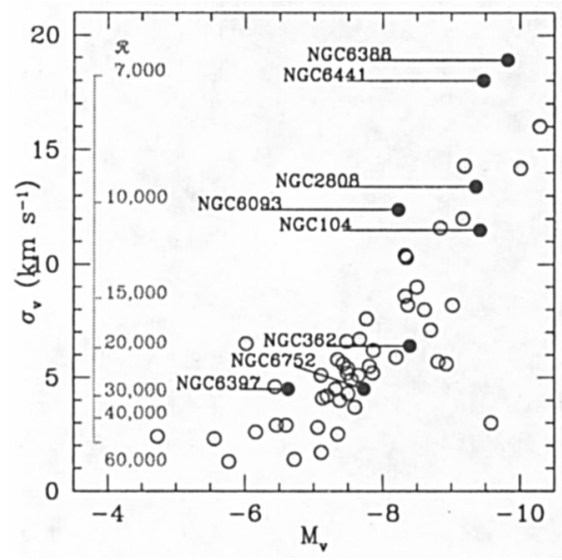

Figure 1. The distribution of Galactic GCs in absolute magnitude $\left(M_{V}\right)$ vs. velocity dispersion $\left(\sigma_{v}\right)$ from Pryor \& Meylan 1993. The in-set scale indicates the spectral resolution $(\mathrm{R}=\lambda / \Delta \lambda)$ which can be obtained for a cluster with the indicated value of $\sigma_{v}$. We have observed the labeled clusters.

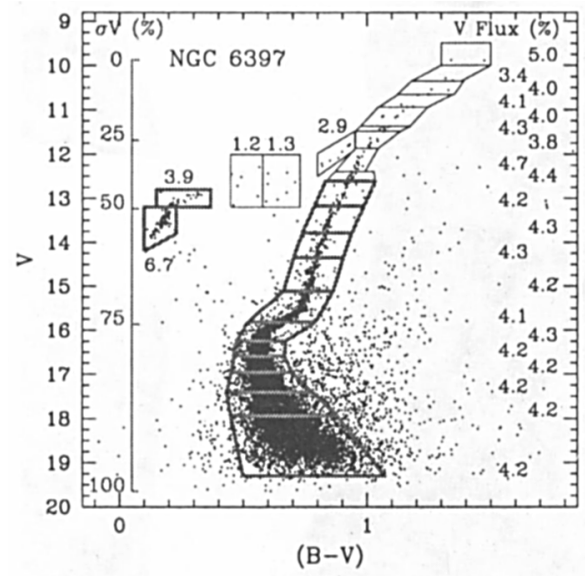

Figure 3. Color-magnitude diagram for NGC 6397 (Kaluzny et al. 1997). The scale on the left shows the cumulative flux from cluster stars with increasing $V$-band flux. The boxes sub-divide the stellar population into groups containing $\sim 5 \%$ of the $V$-band light, as indicated on the right. Thick-lined boxes show populations represented in the central $32 \times 32$ sq. arcsec based on groundbased photometry.

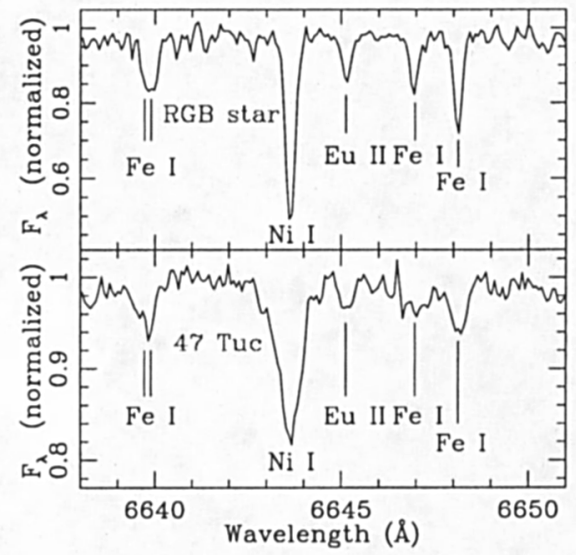

Figure 2. The integrated-light spectrum of 47 Tuc compared with the spectrum of a single RGB star in that cluster. Even with a relatively large $\sigma_{v}(14 \mathrm{~km} / \mathrm{s})$, we can detect absorption lines of the more exotic elements, such as the r-process element Eu.

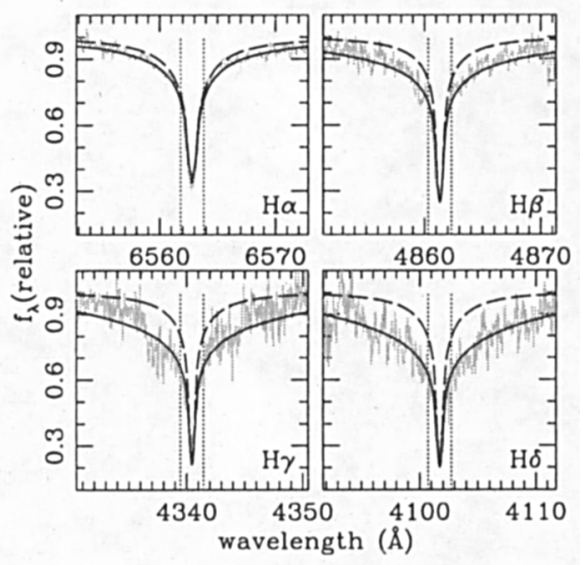

Figure 4. Model Balmer line profiles compared with the observed integrated light spectrum of NGC 6397. Thick lines show model profiles constructed from stellar populations included in the observations (see Figure 3). Dashed lines show model profiles which result from excluding horizontal branch stars. This illustrates that the Balmer line profiles contain clues to the population mix of the cluster. 


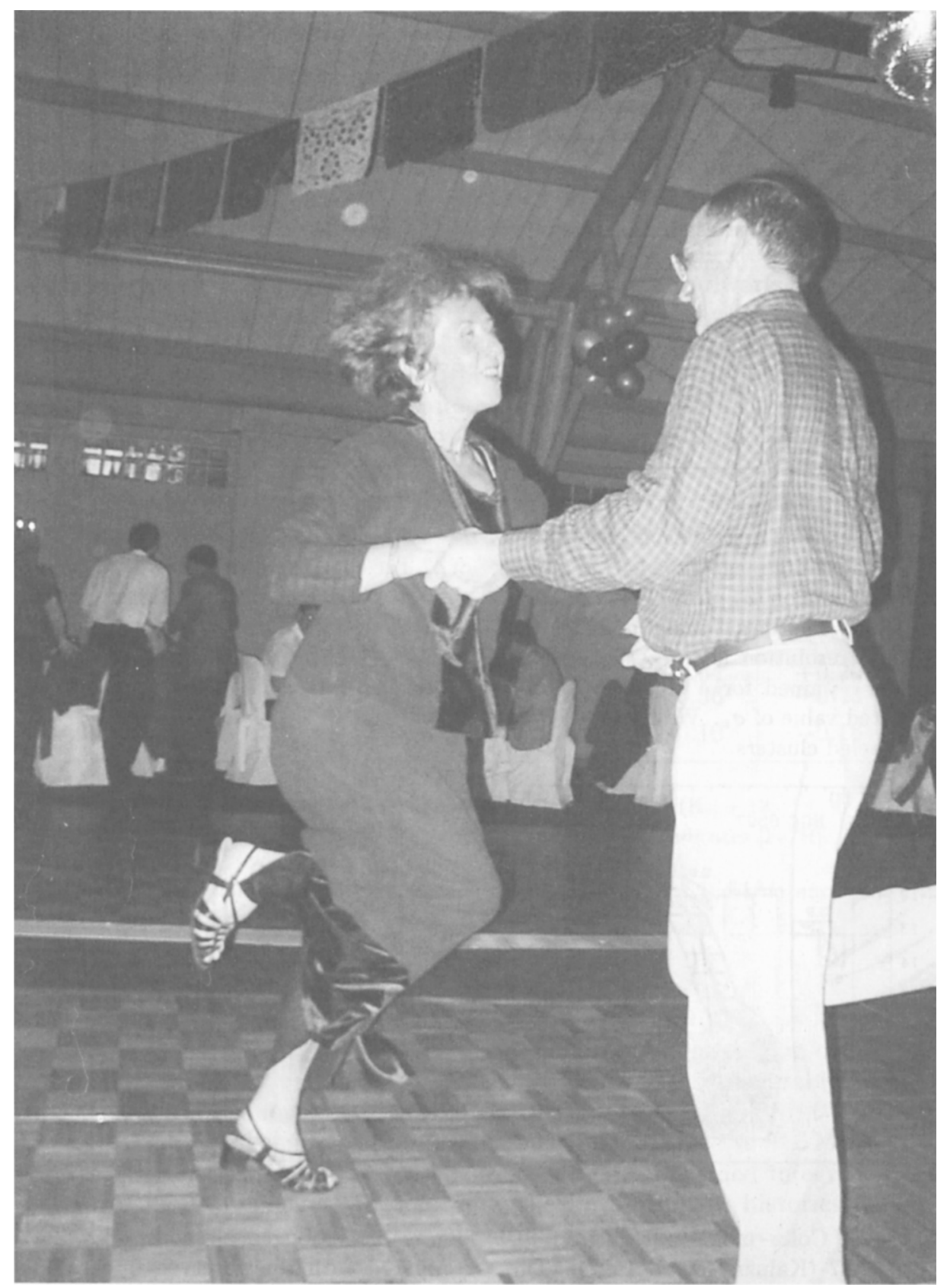

Danielle Alloin and Georges Meylan show how the French do it. 